\title{
Coupled logistic carrying capacity model
}

\author{
H. Mohd Safuan ${ }^{1} \quad$ I. N. Towers ${ }^{2} \quad$ Z. Jovanoski ${ }^{3}$ \\ H. S. Sidhu ${ }^{4}$
}

(Received 13 December 2011; revised 3 May 2012)

\begin{abstract}
This study proposes a coupled nonlinear system based on the logistic equation that models the interaction of a population with its time varying environment. The model eliminates the need for a priori knowledge of the environmental carrying capacity or constraints to be placed upon the initial conditions. Analysis and computer simulations are presented to illustrate the system's dynamical behaviour.
\end{abstract}

\section{Contents}

1 Introduction

2 Modified logistic models

3 Coupled logistic carrying capacity model

http://journal.austms.org.au/ojs/index.php/ANZIAMJ/article/view/4972 gives this article, (C) Austral. Mathematical Soc. 2012. Published June 6, 2012. ISSN 1446-8735. (Print two pages per sheet of paper.) Copies of this article must not be made otherwise available on the internet; instead link directly to this URL for this article. 


\section{Introduction}

Growth models such as the logistic equation have been widely studied and applied in population and ecological modelling. Introduced in the 19th century, the logistic equation describes population growth with a self-limitation term which serves as a correction to the unlimited growth of the Malthusian model. The classical logistic (or Verhulst's) equation is [1]

$$
\frac{\mathrm{dN}}{\mathrm{dt}}=\mathrm{rN}\left(1-\frac{\mathrm{N}}{\mathrm{K}}\right), \quad \mathrm{N}(0)=\mathrm{N}_{0}
$$

where $N(t)$ denotes the population density, $r$ is the intrinsic growth rate, $\mathrm{K}$ is the carrying capacity, and $\mathrm{N}_{0}$ is the initial population density at time $t=0$. The nature of this equation is that the property of the solutions, for all strictly positive initial conditions, approach the constant value carrying capacity, $\mathrm{K}$, as time, $\mathrm{t}$, tends to infinity.

The carrying capacity is usually regarded as a constant, which is not often realistic. Many studies have discussed the importance of time dependent carrying capacities [1]. By using a time dependent carrying capacity, $\mathrm{K}(\mathrm{t})$, the logistic equation is in the form of a nonautonomous function. As opposed to the classical logistic equation for which an exact solution can be found, the analytical solution of the nonautonomous equation is often not possible. In addition, phase plane analysis cannot be utilized if the system is no longer autonomous. For some cases where the system may remain autonomous but not solvable exactly, numerical computations can be used as another option to understand the system. The time dependent forms, $K=K(t)$, have been successfully used to describe the enrichment of a lake by a nutrient [2], to forecast product life cycles of electrical goods [3], and to describe seasonal 
environments [4]. Recently, a functional form of carrying capacity was used to describe the changing micro-environment beneath an occlusion on healthy human skin [5]. Meyer [6] and Meyer and Ausubel [7] used a logistically varying carrying capacity to model the technological development of a human population. For these applications it is important that the carrying capacity is not treated as a constant. Positive changes in environment such as new resources or food production elevates the carrying capacity, whereas a negative change such as the advent of a toxic environment or food depletion will degrade the carrying capacity.

This article links the changing carrying capacity with the logistic equation as a coupled nonlinear differential system. We develop a logistic-type growth model that predicts the changes in the population due to the changes in the environment's carrying capacity.

\section{Modified logistic models}

Several examples exist of a logistic-type equation coupled to an equation for the carrying capacity. Huzimura and Matsuyama [8] studied a model where the rate of change of the carrying capacity decays proportionally to the population size. Another model that tried to link the direct dependency of a population to its carrying capacity was proposed by Thornley and France [9], later modified by Thornley et al. [10], can be written as

$$
\begin{aligned}
& \frac{d N}{d t}=a N\left(1-\frac{N}{K}\right), \\
& \frac{d K}{d t}=-b(K-N),
\end{aligned}
$$

where the positive constants $\mathrm{a}$ and $\mathrm{b}$ are the population growth rate and environmental development rate respectively. As the parameter $b$ is positive it describes the natural loss of carrying capacity but at the same time allows the population to contribute to the carrying capacity itself. An important feature 
of the model is that the asymptotic value of $K(t)$ is not known a priori as it is in the logistic equation (1) where $\mathrm{K}$ is a fixed parameter. However, the model has infinitely many stationary solutions along the line $\mathrm{N}=\mathrm{K}$. Therefore initial conditions $\mathrm{N}(0)=\mathrm{K}(0)$ will have no dynamical behaviour which is unfortunate for the case where the population is initially in equilibrium with its environment. Effectively, within this model, the choice of initial condition determines the asymptotic carrying capacity. System (2) can be rescaled using a set of substitutions $n=b N, k=b K, \tau=a t$, such that

$$
\begin{aligned}
& \frac{d n}{d \tau}=n\left(1-\frac{n}{k}\right), \\
& \frac{d k}{d \tau}=-\gamma(k-n),
\end{aligned}
$$

where $\gamma=\mathrm{b} / \mathrm{a}$ is a ratio between the environmental development rate and the population growth rate. The system (3) has steady states at $n^{*}=k^{*} \neq 0$ which is an infinite line. ${ }^{1}$ The line $n^{*}=k^{*} \neq 0$ is a stable critical line with eigenvalues of $\lambda_{1,2}=0,-(\gamma+1)$ of the Jacobian matrix. With zero and negative real eigenvalues, all trajectories in the first quadrant $(n, k>0)$ will approach the critical line for a given set of initial conditions. The system shows that by providing specific values of initial conditions, the solution will always change to another stable solution (see curves (i) and (ii) in Figure 1a). When initial conditions start at the same value, for example $n_{0}=k_{0}=0.1$, no growth occurs for both population and its environment, hence they remain the same for all time. This can be seen in curve (iii) in Figure 1a. The system requires the initial value of the carrying capacity to be a higher value than the initial population in order to observe the population's growth. If the initial carrying capacity is lower than the initial population, the population decreases toward the carrying capacity. Figure $1 \mathrm{~b}$ demonstrates the effect of different parameter values for $\gamma$ for fixed initial conditions with $k_{0}>n_{0}$.

${ }^{1} \mathrm{k}=0$ is singular in the differential equations (3). Note that by cancellation of the common factor $(k-n)$ the system (3) has $d n / d k=-n /(\gamma k)$ and hence trajectories, $n \propto k^{-1 / \gamma}$, are hyperbolic-like. 


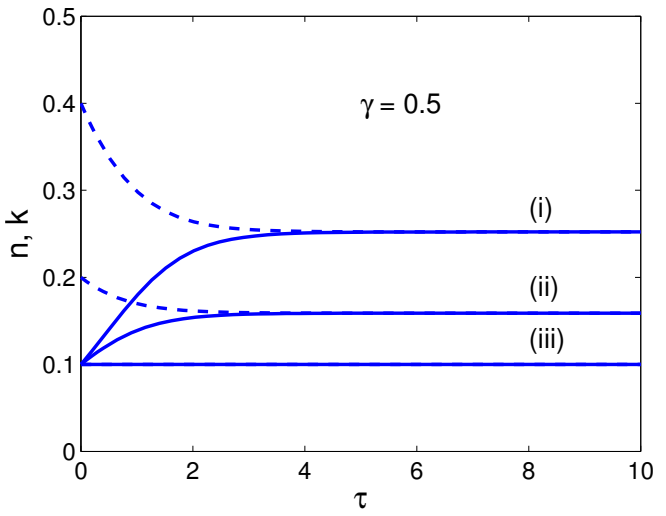

(a)

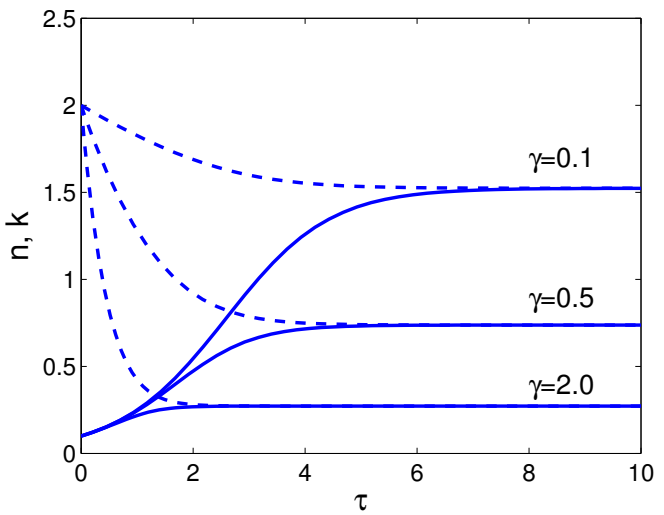

(b)

Figure 1: Logistic growth curve (solid line) and its carrying capacity (dashed line) in system (3) for different initial values (a) $\gamma=0.5$ and $n_{0}=0.1$ with (i) $k_{0}=0.4$, (ii) $k_{0}=0.2$, (iii) $k_{0}=0.1$, (b) $\gamma=0.1,0.5$, 2.0 with $\left(n_{0}, k_{0}\right)=(0.1,2.0)$.

\section{Coupled logistic carrying capacity model}

Unlike the models proposed by Huzimura and Matsuyama [8] and Thornley and France [9], we introduce a modified coupled logistic carrying capacity model that could incorporate the dependency of a population and its environment by using a nonlinear interaction term. We start by assuming a standard logistic equation for the population $\mathrm{N}$ with constant growth rate $\mathrm{a}$. The carrying capacity $\mathrm{K}$ is assumed to change at a rate proportional to its present value with proportionality constant $b$, the development rate. The population depletes the carrying capacity through an interaction term with the rate $\mathbf{c}$. Instead of the population independently contributing to the development of the carrying capacity, our model encompasses the dependency of the carrying capacity to change through an interaction with the population. Thus we 
modify the Thornley model (2) to

$$
\begin{aligned}
& \frac{d N}{d t}=a N\left(1-\frac{N}{K}\right), \\
& \frac{d K}{d t}=b K-c K N .
\end{aligned}
$$

Meyer [6] and Meyer and Ausubel [7] introduced the carrying capacity function as $\mathrm{dK} / \mathrm{dt}=\alpha \mathrm{K}-\alpha \mathrm{K}^{2} / \mathrm{K}_{\alpha}$ in their models. The nonlinear term $\mathrm{K}^{2}$ ensures the carrying capacity is self-limiting. If the population influences the carrying capacity or vice versa, this can be viewed as an interspecific relation between the population and its carrying capacity. Thus, in our model, we introduce the nonlinear term $\mathrm{KN}$ to represent this interaction in Equation (4b). Equation (4b) can also be written as $d K / d t=b K(1-\alpha N)$ where $\alpha=c / b$. By introducing non-dimensionalized parameters

$$
\mathrm{n}=\alpha \mathrm{N}, \quad \mathrm{k}=\alpha \mathrm{K}, \quad \text { and } \quad \tau=\mathrm{at},
$$

we obtain the non-dimensionalized form of the model (4)

$$
\begin{aligned}
& \frac{d n}{d \tau}=n\left(1-\frac{n}{k}\right), \\
& \frac{d k}{d \tau}=\mu k(1-n),
\end{aligned}
$$

where $\mu=b / a$ is a ratio between the carrying capacity development rate and the population growth rate. By equating the system (5) to zero, one steady state is obtained at $\left(n^{*}, k^{*}\right)=(1,1) .^{2}$ To examine the stability properties in the neighbourhood of the steady state, we use the Jacobian matrix

$$
J=\left(\begin{array}{cc}
1-\frac{2 n}{k} & \frac{n^{2}}{k^{2}} \\
-\mu k & \mu(1-n)
\end{array}\right) .
$$

${ }^{2}$ As before $k=0$ is singular in the system (5). 
For the steady state, the Jacobian's eigenvalues are $\lambda_{1,2}=(-1 \pm \sqrt{1-4 \mu}) / 2$. Hence the point $(1,1)$ is a stable spiral if $\mu>1 / 4$, or a stable node otherwise. The system (3) is autonomous and can be solved exactly. However, for our model (5) only numerical solutions can be obtained due to the nonlinearity of the system. An advantage of system (5) is that there exists a unique stable attractor for any initial conditions $(n, k)=\left(n_{0}, k_{0}\right)$. Any selection of $n_{0}$ and $k_{0}$ will approach the same equilibrium solution. We start from the first case where the population and its carrying capacity are at the same level, $n_{0}=k_{0}$. Figure 2 shows the behaviour of solutions of $n$ and $k$ using the model (5). When $\mu>1 / 4$, oscillations between $n$ and $k$ are expected as demonstrated by the linear stability analysis. Overshoots for $n$ and $k$ can be seen and are more pronounced if the value of $\mu$ is much greater than $1 / 4$.

For $\mu=2(b=2 a)$, the development rate is double the growth rate and as a result, the carrying capacity shows a rapid increase initially while a slower growth in the population. This increase in the carrying capacity, indicative of an improved environment, is more conducive for further population growth. At the stage when the carrying capacity depletes due to the interaction with the population, the population eventually will have to fall. Moreover, an overpopulated environment exhausts available resources thus lowering the carrying capacity. With the population losses, the resource becomes richer once more which allows the population to slowly grow back, and exhibits repeated (damped) oscillations until the population reaches the stable environment.

Figure $2 \mathrm{~b}$ illustrates that if the population growth rate is equal to its carrying capacity development rate, $(\mu=1, b=a)$, then the difference between $\mathrm{n}$ and $\mathrm{k}$ in the early stage of growth is smaller compared to when $\mu=2$. A small difference can be seen when $\mu=0.5$ in Figure $2 \mathrm{c}$ with the carrying capacity growing slightly faster than that of the population. As $\mu$ gets smaller $(\mu=0.2<1 / 4)$, the population and the carrying capacity gradually increase to the equilibrium level (Figure $2 \mathrm{~d}$ ).

For the second case when an initially rich environment is assumed, a huge 
increase in the carrying capacity can be seen, followed by an increase in the population as shown in Figure 3. Once the carrying capacity shows depletion, the population density also starts to decline. We observe in the figure that the variation in the carrying capacity is very large compared to the variation in the population growth. For example, for $\mu=2$ Figure 3 a shows that the carrying capacity peaks at about 36 before decaying, but when $\mu=0.2$ Figure 3d shows that the carrying capacity peak is less than 12 times smaller than for $\mu=2$. In contrast with the cases in Figure 2c and 2d where there were only small numerical differences between the population and the carrying capacity, Figure 3 shows more pronounced variations between the population and the carrying capacity caused by their initial values.

\section{Discussions}

The concept of the carrying capacity as the environment's maximal load defines the limited growth of a population. The difficulty of determining the exact form of the carrying capacity often means certain assumptions have to be made. The contribution of environmental enrichment to the carrying capacity, due to an increase of nutrients or food resources, is one possible assumption. Since there is no specific way to model the carrying capacity, we proposed the modified logistic growth model. The model encompasses the direct dependency of the growth equations with its carrying capacity. Instead of assuming the carrying capacity as an independent parameter, our model provides the physical idea of the inter-relation that coexists between the environment and the population. By connecting them, we understood the effect of a varying carrying capacity on the population's growth.

Our results show that development of the carrying capacity encourages the population to grow. The main parameters in our model (4) are determined by the population growth rate, $a$, and the development rate, $b$, of the carrying capacity. Oscillations occur in the growth curves by fixing the carrying capacity development rate to be more than a quarter of the population 


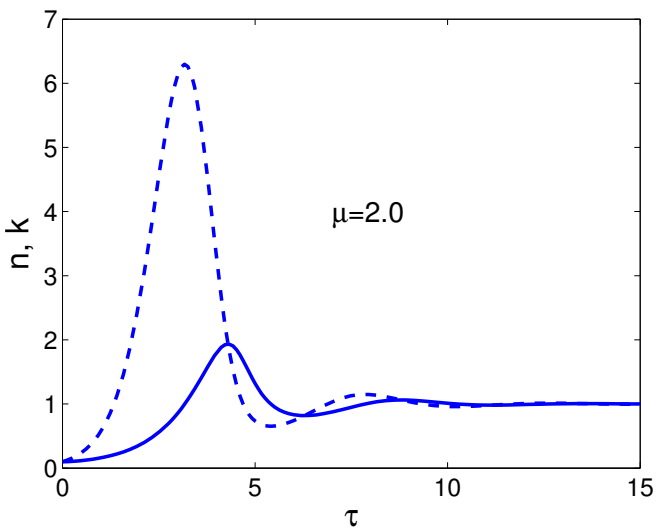

(a)

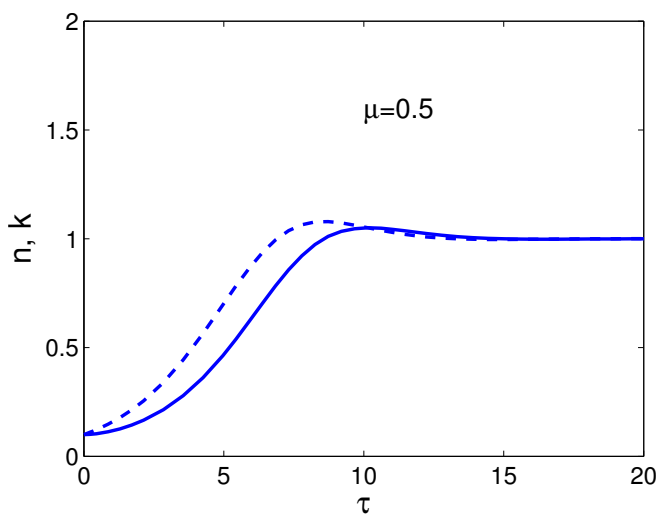

(c)

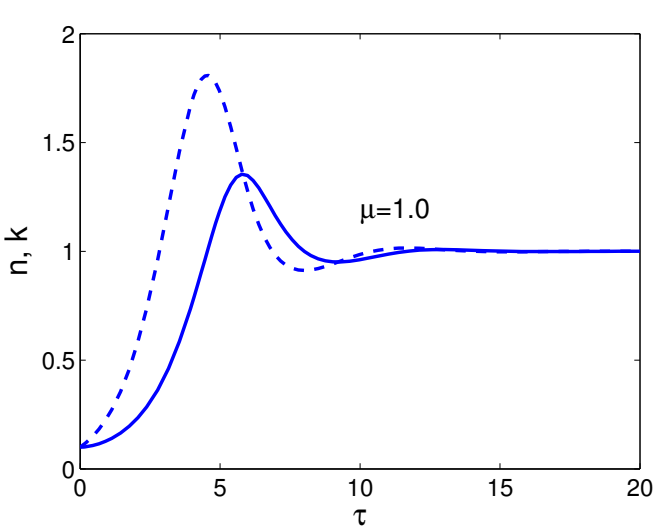

(b)

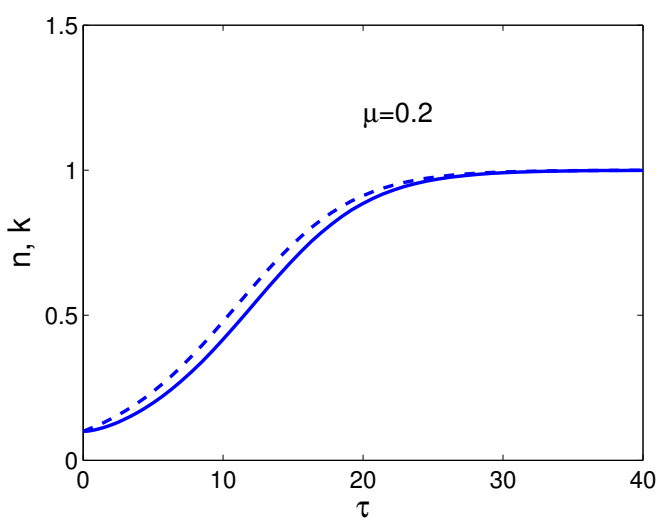

(d)

Figure 2: Logistic growth curve (solid line) and its carrying capacity (dashed line) in system (5) with (a) $\mu=2.0$, (b) $\mu=1.0$, (c) $\mu=0.5$, and (d) $\mu=0.2$ with $n_{0}=k_{0}=0.1$ (note that different scales are used). 


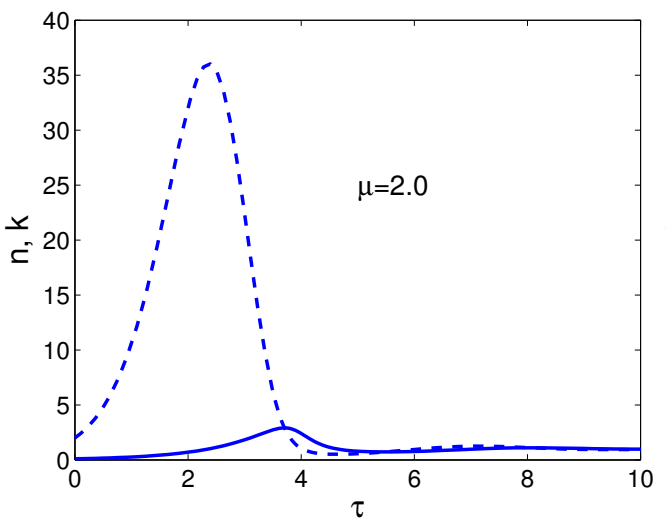

(a)

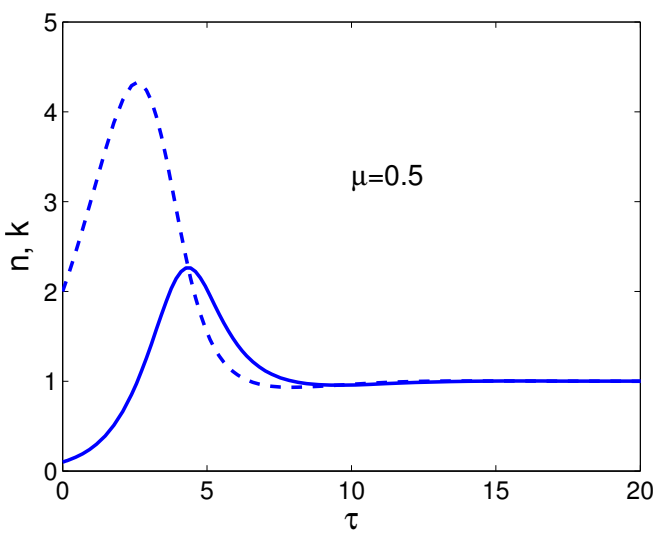

(c)

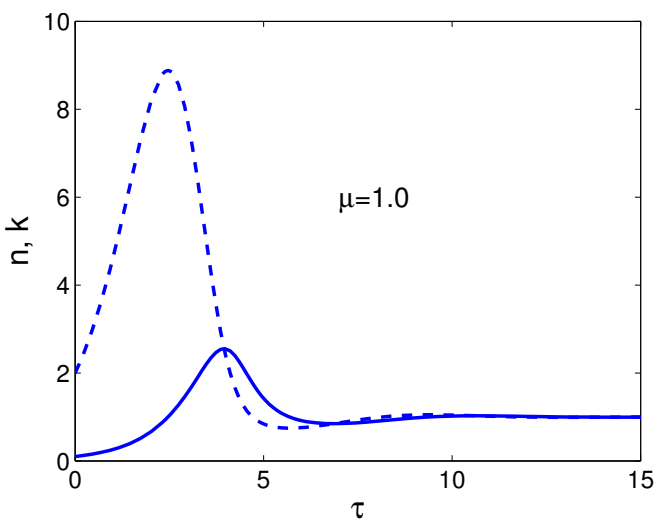

(b)

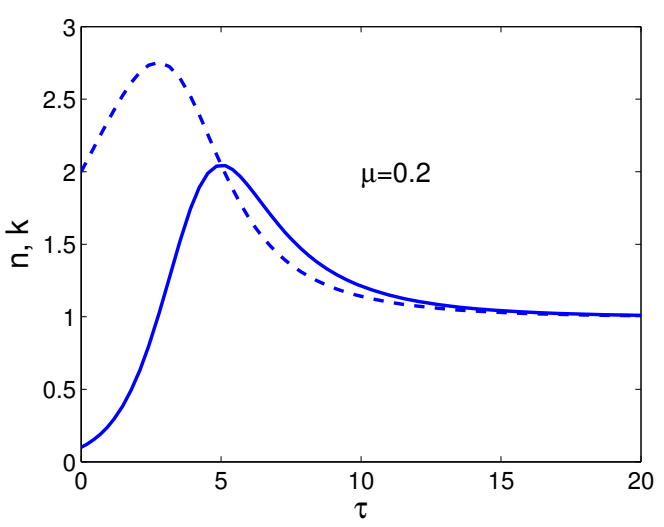

(d)

Figure 3: Logistic growth curve (solid line) and its carrying capacity (dashed line) in system (5) with (a) $\mu=2.0$, (b) $\mu=1.0$, (c) $\mu=0.5$, and (d) $\mu=0.2$ with $n_{0}=0.1, k_{0}=2.0$ (note that different scales are used). 
growth rate. Our model is an extension of previous modified logistic models, and is still open for further exploration. Our work only considered a simple interaction term between the population and its environment via the carrying capacity. Other forms of interaction terms are also possible depending on particular cases. The model has the potential to be used as an application in biological and ecological sciences.

Acknowledgements HMS thanks Universiti Tun Hussein Onn Malaysia, School of Physical, Environmental and Mathematical Sciences, UNSW Canberra and ANZIAM/CSIRO Student Support Scheme for financial support.

\section{References}

[1] R. B. Banks. Some Basic Frameworks. In F. John, J. E. Marsden, L. Sirovich, M. Golubitsky and W. Jäger (Eds.). Growth and Diffusion Phenomena:Mathematical Frameworks and Applications, 5-6, Springer-Verlag, Berlin, Germany, 1994. C173

[2] S. Ikeda and T. Yokoi. Fish population dynamics under nutrient enrichment-A case of the East Seto Inland Sea. Ecological Modelling, 10, 141-165, 1980. doi:10.1016/0304-3800(80)90057-5 C173

[3] C. V. Trappey and H. Y. Wu. An evaluation of the time-varying extended logistic, simple logistic, and Gompertz models for forecasting short product lifecycles. Advanced Engineering Informatics, 22, 421-430, 2008. doi:10.1016/j.aei.2008.05.007 C173

[4] S. P. Rogovchenko and Y. V. Rogovchenko. Effect of periodic environmental fluctuations on the Pearl-Verhulst model. Chaos, Solitons and Fractals, 39, 1169-1181, 2009. doi:10.1016/j.chaos.2007.11.002 C174

[5] H. Safuan, I. N. Towers, Z. Jovanoski and H. S. Sidhu. A simple model for the total microbial biomass under occlusion of healthy human skin. 
In Chan, F., Marinova, D. and Anderssen, R.S. (eds) MODSIM2011, 19th International Congress on Modelling and Simulation. Modelling and Simulation Society of Australia and New Zealand., 733-739, 2011. C174

[6] P. Meyer. Bi-logistic Growth. Technological Forecasting and Social Change, 47, 89-102, 1994. doi:10.1016/0040-1625(94)90042-6 C174, $\mathrm{C} 177$

[7] P. Meyer and J. H. Ausubel. Carrying Capacity: A Model with Logistically Varying Limits. Technological Forecasting and Social Change, 61, 209-214, 1999. doi:10.1016/S0040-1625(99)00022-0 C174, $\mathrm{C} 177$

[8] R. Huzimura and T. Matsuyama. A mathematical model with a modified logistic approach for singly peaked population processes. Theoretical Population Biology, 56, 301-306, 1999. doi:10.1006/tpbi.1999.1426 C174, C176

[9] J. H. M. Thornley and J. France. An open-ended logistic-based growth function. Ecological Modelling, 184, 257-261, 2005.

doi:10.1016/j.ecolmodel.2004.10.007 C174, C176

[10] J. H. M. Thornley, J. J. Shepherd and J. France. An open-ended logistic-based growth function: Analytical solutions and the power-law logistic model. Ecological Modelling, 204, 531-534, 2007. doi:10.1016/j.ecolmodel.2006.12.026 C174

\section{Author addresses}

1. H. Mohd Safuan, Applied and Industrial Mathematics Research Group, School of Physical, Environmental and Mathematical Sciences, University of New South Wales, Canberra 2600, Australia. mailto:hamizah.mohdsafuan@student . adfa.edu . au 
2. I. N. Towers, Applied and Industrial Mathematics Research Group, School of Physical, Environmental and Mathematical Sciences, University of New South Wales, Canberra 2600, Australia. mailto: i.towers@adfa.edu.au

3. Z. Jovanoski, Applied and Industrial Mathematics Research Group, School of Physical, Environmental and Mathematical Sciences, University of New South Wales, Canberra 2600, Australia. mailto:z.jovanoski@adfa.edu.au

4. H. S. Sidhu, Applied and Industrial Mathematics Research Group, School of Physical, Environmental and Mathematical Sciences, University of New South Wales, Canberra 2600, Australia. mailto:h.sidhu@adfa.edu.au 